\title{
Scalar Structure Underlies Telicity in "Degree Achievements"
}

\author{
Jennifer Hay, Christopher Kennedy and Beth Levin \\ Northwestern University
}

\section{The Aspectual Characteristics of Degree Achievements}

So-called "degree achievements" (DAs), such as widen, lengthen, cool, dry, and straighten, have long caused problems for theories of aspectual classification, because they constitute one area in which the traditional Vendler/Dowty system breaks down. ${ }^{1}$ As first observed in Dowty 1979, these verbs display both telic and atelic properties according to standard diagnostics (see also Abusch 1986). This paper explores and analyzes the specific problems associated with DAs in an effort to better understand the underpinnings of telicity.

The aspectual inconsistency of DAs can be illustrated by examining their behavior with respect to several standard tests for (a)telicity. For example, atelic predicates are known to be entailed by their progressive forms, while telic predicates are not (Vendler 1957, Dowty 1979):
a. Kim is singing. $\Rightarrow$ Kim has sung.
b. $\quad \mathrm{Kim}$ is writing a song. $\nRightarrow \mathrm{Kim}$ has written a song.

An examination of the examples in (2) shows that different DAs behave differently with respect to this test. The verb lengthen appears to display atelic behavior, whereas the verb straighten appears to be telic.

(2) a. Kim is lengthening the rope. $\Rightarrow$ Kim has lengthened the rope.

b. Kim is straightening the rope. $\nRightarrow$ Kim has straightened the rope.

A second test indicates that individual DAs also appear to display ambiguous telicity. As observed in Vendler 1957 and Dowty 1979, for-adverbials modify only atelic predicates, while in-adverbials occur only with telic predicates:

(3) a. Pug snoozed for/?in an hour.

b. Pug destroyed the couch ?for/in an hour.

Many DAs, however, can appear with both for-adverbials and in-adverbials, as illustrated by the examples with cool in (4) (cf. Dowty 1979).

(4) a. The soup cooled for an hour.

b. The soup cooled in an hour. 
A third test confirms the second's results, and further shows that the telicity of a single DA can be influenced by context. As illustrated by (5a), a telic predicate modified by almost is ambiguous between a reading in which the described event is claimed to have occurred, but not quite been completed, and one in which it is asserted not to have occurred at all (Dowty 1979). An atelic predicate modified by almost has only the latter type of reading, however, as illustrated by (5b), which can only mean that Lee came close to reciting poetry, but didn't actually do so.
a. Lee almost read Eugene Onegin.
b. Lee almost recited poetry.

Given the facts in (4), it is not surprising that individual DAs show both telic and atelic behavior with respect to the almost test. What is interesting, however, is that their aspectual properties may be influenced by the contexts in which they appear. For example, real-world knowledge about pants and exams can affect the telicity of different uses of the verb lengthen. In (6a) lengthen is telic, and the sentence is ambiguous, but in (6b), lengthen has an atelic interpretation only, and the sentence is unambiguous.

(6) a. The tailor almost lengthened my pants.

b. The teacher almost lengthened the exam.

The aspectual variability of DAs is reminiscent of that manifested by some other verbs in combination with mass vs. count noun arguments. As is well known (Declerck 1979, Dowty 1979, Krifka 1989, 1992, Verkuyl 1972), certain verbs show telic interpretations with count noun objects and atelic interpretations with mass noun objects, as in (7).

(7) a. Pat is eating a plum. $\nRightarrow$ Pat has eaten a plum.

b. Pat is eating rice. $\Rightarrow$ Pat has eaten rice.

This effect has been analyzed in terms of the nature of what we refer to as the "affected" argument-the argument that undergoes a change as a result of the action described by the verb (see Dowty 1991, Jackendoff 1996, Krifka 1989, 1992, Tenny 1987, 1994, Verkuyl 1972, 1993, Ramchand 1997). Count nouns denote a bounded quantity of stuff, supporting the identification of a terminal point for the event described by the verb, which leads to a telic interpretation. Mass nouns denote a nonbounded quantity of stuff, however, so that no such terminal point can be identified, and an atelic interpretation results.

In order to bring out the parallel between examples like (7) and DAs most effectively, we point out that the affected argument figures in the determination of the telicity of verbs such as eat by virtue of the (non)boundedness of a physical property of the entity it denotes. This point is not to our knowledge made explicit in the formal accounts of the effects of mass-count nouns on telicity, which confuse this property with the argument itself, a point we come back to in section 4.2. This 
physical property is lexically determined by the verb; in (7), for example, the relevant property is volume. When the volume property of the affected argument of eat is bounded, as in (7a), a terminal point can be identified for the event introduced by the verb (the point of consumption of the entire plum), resulting in a telic interpretation. When the volume property is nonbounded, as in (7b) (an unspecified amount of rice cannot be assumed to have a maximal volume), no terminal point can be identified for the event, and the predicate has an atelic interpretation.

The primary goal of this paper is to demonstrate that the variable aspectual properties of DAs can be explained in essentially the same way as those of verbs like eat. A basic semantic characteristic of DAs is that their affected argumentjust like the affected argument of eat-undergoes a change in some property. In deadjectival DAs, the change is in the property associated with the meaning of the adjectival base. We argue that when the degree to which this property changes can be interpreted as bounded, a telic interpretation of the predicate arises, and when the degree of change must be considered nonbounded, an atelic interpretation results. In its broad outlines our analysis resembles the analyses of verbs of change of statethe larger set of verbs that the DAs belong to-in Jackendoff 1996, Ramchand 1997, and Tenny 1994; however, many verbs of change of state do not show the variable telicity of DAs, so much of our effort is devoted to showing how this type of account can handle the full complexity of the (a)telicity of DAs.

Thus, a second goal of the paper is to show that the variable aspectual behavior of many DAs can be explained in terms of the relation between event structure and the scalar structure of gradable properties. Specifically, when the scalar structure associated with the base adjective has a natural bound, the derived verb is telic; when the adjective's scalar structure has no such bound, the verb is atelic. We go beyond previous work by demonstrating that the telicity of a particular DA can be, and often is, derived through a process of conversational implicature, indicating that the aspectual properties of a particular predicate often cannot be completely specified in terms of formal (semantic or syntactic) features (cf. Olsen 1994, 1997). We then show that the analysis presented here has applications beyond the specific class of degree achievements, extending to other types of predicates with similar behavior. Finally, we show that our analysis of degree achievements provides new insights into the relation between verbal aspect and the semantic properties of a verb's arguments. In particular, it suggests a new understanding of the notion of "incremental theme"- the expression whose properties have been claimed to be crucial to determining (a)telicity.

\section{The Lexical Semantics of Degree Achievements}

As noted above, a basic semantic characteristic of DAs is that they describe changes in some property of one of their arguments; in the case of DAs derived from gradable adjectives, this property is associated with the meaning of the base adjective. This feature of DAs can be exploited to provide an account of their aspectual properties that mirrors the analysis of the mass-count distinction illustrated in (7). Specifi- 
cally, we claim that these predicates introduce a measure of the amount to which an argument of the verb-the object in the transitive forms, e.g., (2); the subject in the intransitive forms, e.g., (4)—changes with respect to the gradable property introduced by the adjectival base (cf. Jackendoff 1996, Ramchand 1997, Tenny 1994). We refer to this measure as the "difference value." That is, we claim that the interpretations of the examples in (8) are as paraphrased in (9), where the difference values are italicized.

(8) a. Kim lengthened the rope.

b. Kim lengthened the rope 5 inches.

(9) a. Kim caused the length of the rope to increase by some amount

b. Kim caused the length of the rope to increase by 5 inches

We further claim that the formal properties of the difference value determine the predicate's telicity. When the difference value identifies a bound on the measure of change in the affected argument over the course of the event, the predicate is telic. In contrast, when the difference value does not impose such restrictions, the measure of change is nonbounded, and the predicate is interpreted atelically. In (8a), for example, the implicit difference value, represented as an indefinite amount of change in $(9 a)$, provides no bound on the measure of change undergone by the affected argument (the rope), and the predicate is atelic. In contrast, the measure phrase in (8b) introduces a bounded measure of change, represented by the definite amount of change in (9b). As shown by the failure of the entailment in (10), the result is a telic interpretation.

(10) Kim is lengthening the rope 5 in. $\not \Rightarrow \mathrm{Kim}$ has lengthened the rope 5 in.

In section 3, we show that this proposal, together with a more detailed picture of the semantic properties of gradable adjectives and the contributions of contextual information, can be extended to an account of the full range of facts introduced in section 1. Before turning to this discussion, we show how the lexical semantic analysis of DAs sketched here can be formalized.

Since a large number of DA are derived from gradable adjectives, we begin by outlining our basic assumptions about their semantics. (We return to other types of verbs in section 4.1.) We follow Kennedy 1999 in analyzing gradable adjectives as functions from objects to abstract representations of measurement, or degrees (see also Bartsch and Vennemann 1973). To reflect the fact that the same object may manifest different degrees of the same gradable property at different times, we extend this analysis by incorporating a temporal variable, so that the denotation of an expression of the form long $(x)(t)$ is as in (11).

$\llbracket \operatorname{long}(x)(t) \rrbracket=$ the degree to which $x$ is long at time $t$

Degrees themselves are formalized as positive or negative intervals on a scale (Seuren 1978, von Stechow 1984b, Kennedy 1997), where a scale is a set of 
points totally ordered along some dimension (e.g., TEMPERATURE, BRIGHTNESS, LENGTH, VOLUME, etc.). This distinction supports a theory of adjectival polarity in which positive adjectives like long and wide are analyzed as functions from objects to positive degrees, and negative adjectives like short and narrow denote functions from objects to negative degrees. (See Kennedy 1999 for extensive justification of this approach.) The sets of positive and negative degrees on a scale $S$ is formally defined in (12), and the basic idea is illustrated in (13), which indicates the positive and negative projections of some object $x$ on a scale $S$ at time $t$.

$$
\begin{array}{ll}
\text { a. } & P O S(S)=\left\{d \subseteq S \mid \exists p_{1} \in d \forall p_{2} \in S\left[p_{2} \preceq p_{1} \rightarrow p_{2} \in d\right]\right\} \\
\text { b. } & N E G(S)=\left\{d \subseteq S \mid \exists p_{1} \in d \forall p_{2} \in S\left[p_{1} \preceq p_{2} \rightarrow p_{2} \in d\right]\right\} \\
S: & 0 \\
& \operatorname{pos}(x)(t) \longrightarrow \infty
\end{array}
$$

What is important for our purposes is that this model supports a straightforward characterization of "degree addition" (see von Stechow 1984b), which we use to formalize the hypothesis that the lexical meaning of a DA includes a specification of the amount to which some object increases in the degree to which it possesses some gradable property. Assume first that every point on a scale can be associated with a numerical value in the set of real numbers. Positive and negative degrees can then be labeled as in (14), where (14a) denotes the positive degree ranging from the lower bound of the scale to point $i$, and (14b) denotes the negative degree ranging from point $i$ to the upper end of the scale, which we denote with the $\infty$ symbol to indicate the fact that scales typically have no maximal element (see von Stechow 1984a, Rullmann 1995; this assumption is revised in section 3.2 below).

$$
\begin{array}{ll}
\text { a. } & d_{\langle 0, i\rangle} \\
\text { b. } & d_{\langle i, \infty\rangle}
\end{array}
$$

Using representations like those in (14), degree addition can be defined as in (15).

$$
\begin{array}{ll}
\text { a. } & d_{\langle 0, i\rangle}+d_{\langle 0, j\rangle}=d_{\langle 0, i+j\rangle} \\
\text { b. } & d_{\langle i, \infty\rangle}+d_{\langle 0, j\rangle}=d_{\langle i-j, \infty\rangle}
\end{array}
$$

Note that these definitions define degree addition only for two positive degrees or a negative and a positive degree; addition of two negative degrees is undefined. (For theoretical and empirical justification of this position, see von Stechow 1984b.) Adding the final assumption that measure phrases denote positive degrees (von Stechow 1984b), we are in a position to move to the formal analysis of DAs.

The lexical semantic analysis of DAs informally presented above claims that DAs describe events that measure out the change an object undergoes with respect to the gradable property introduced by the base adjective. This analysis can be implemented by introducing a function INCREASE, which we assume to be contributed by the verb-forming - en $\$$ morpheme, which takes a gradable adjective meaning $\phi$ and returns a description of an event of some object undergoing a change in the degree to which it is $\phi$. The proposal is made explicit in the truth conditions in (16), where SPO and EPO are functions from events to times that return an event's beginning and end points, respectively. 


$$
\llbracket \operatorname{INCREASE}(\phi)(x)(d)(e) \rrbracket=1 \text { iff } \phi(x)(\operatorname{SPO}(e))+d=\phi(x)(\operatorname{EPO}(e))
$$

In prose, INCREASE $(\phi)(x)(d)$ is true of an event $e$ just in case the degree to which $x$ is $\phi$ at the beginning of the event plus $d$ equals the degree to which $x$ is $\phi$ at the end of the event; i.e., just in case $x$ increases in $\phi$-ness by $d$. This measure of change corresponds to what we have called the difference value.

The logical representation in (16) is intended to capture the core meaning of all DAs-both the transitive and intransitive forms. An important difference between the two forms is that the former contains a causative component that the latter appears to lack. However, since the exact analysis of this element of meaning is not central to the topics of this paper, we leave it aside here. ${ }^{2}$ For simplicity, we omit the external argument and causative component from the logical representations of transitive examples in the following discussion. We should point out, however, that the fact that both transitive and intransitive DAs show the same sort of variable aspectual behavior-they can be either telic or atelic-indicates that causation must be kept separate from (a)telicity, reaffirming arguments by Abusch 1986, Pustejovsky 1991, Rappaport Hovav and Levin 1999, and Van Valin and LaPolla 1997. (See McCawley 1976 for early observations supporting the independence of causativeness and aspectual classification and Van Valin and LaPolla 1997 for examples of lexical causatives belonging to all aspectual classes.)

As an illustration of the analysis, consider (17), which provides (the relevant parts of) the logical representations of (8a) and (8b) (assuming existential closure over free variables).

$$
\begin{aligned}
& \text { a. } \quad \exists e, d[\operatorname{INCREASE}(\text { long }(\text { rope }))(d)(e)] \\
& \text { b. } \quad \exists e[\operatorname{INCREASE~}(\text { long }(\text { rope }))(5 \text { inches })(e)]
\end{aligned}
$$

The truth conditions associated with these expressions are identical to the paraphrases of the original sentences' meanings in (9a) and (9b). Ignoring the causative component, (8a) is true just in case the length of the rope at the end of the increasing event equals its length at the beginning plus some unspecified degree of length. (8b) is true just in case the length of the rope at the end of the increasing event equals the length at the beginning plus the degree of length denoted by 5 inches.

Our analysis differs from some previous accounts in an important way. A common approach to the problem of the variable aspectual behavior of DAs has been to postulate a systematic ambiguity in these verbs' meanings (see Abusch 1986, Declerck 1979, Bertinetto and Squartini 1995). On this view, telic interpretations correspond to lexical semantic representations of the form [BECOME $(\operatorname{adj}(x))]$, and atelic interpretations are associated with semantic representations that have the form [BECOME $(a d j-e r(x))]$, where adj-er represents the comparative form of the base adjective. In contrast, our analysis does not introduce an ambiguity in the semantic representation of degree achievements. As we demonstrate in the next section, the (a)telicity of degree achievements can be fully explained in terms of the semantic properties of the difference value: a DA is telic when the difference value is bounded and atelic when it is non-bounded. Since there is no independent 
evidence for a systematic ambiguity in these verbs, an account that does not rely on such an ambiguity should be preferred to one that does.

\section{Telicity and the Difference Value}

Recall from section 1 that the explanation of the effect of the mass-count noun distinction on telicity is that the boundedness of the volume property of a count noun supports the identification of an endpoint for the event described by the verb, whereas the absence of such a bound for the volume property of a mass noun makes the identification of an endpoint for the event impossible. The lexical semantic analysis in section 2 supports exactly the same type of analysis of the aspectual properties of DAs. Crucial to this analysis is the difference value. If the difference value specifies a bounded amount of change in the degree to which the affected argument possesses the gradable property identified by the base adjective, then a terminal point for the entire event can be identified. The endpoint is that point at which the affected argument possesses a degree of the measured property that equals the initial degree to which it possessed this property plus the degree denoted by the difference value. If, however, the difference value does not specify a bounded measure of change, then this computation cannot be performed, and no terminal point can be identified. Our analysis thus predicts that a DA should have a telic interpretation when the difference value corresponds to a bounded measure of change, and an atelic interpretation when the difference value does not identify a bounded measure of change. In the following sections, we consider the full range of DA phenomena, showing that the facts bear out our predictions exactly.

\subsection{Specifying the Difference Value}

The most straightforward DA constructions are those in which overt linguistic material places a bound on the difference value. This can be accomplished in two ways. In the first type of case, a measure phrase explicitly identifies a bounded measure of change, as in (18).

(18) a. They widened the road $5 \mathrm{~m}$.

b. The lake cooled 4 degrees.

The prediction of our analysis is that such examples should be telic. In (18b), for example, the endpoint of the cooling event can be associated with the point in time at which the degree to which the lake is cool has increased by 4 degrees. The sentences in (19)-(20) verify that (18a) and (18b) are telic.

(19) a. They are widening the road $5 \mathrm{~m} . \not \Rightarrow$ They have widened the road $5 \mathrm{~m}$.

b. $\quad$ The lake is cooling 4 degrees. $\not \Rightarrow$ The lake has cooled 4 degrees. 
(20) a. They almost widened the road $5 \mathrm{~m}$. (AMBIGUOUS)

b. The lake almost cooled $4 \mathrm{deg}$. (AMBIGUOUS)

The second way in which the difference value can receive a bounded interpretation is through the use of degree modifiers that make explicit reference to an endpoint, such as completely, as in (21a) and (21b).

(21) a. They straightened the rope completely.

b. The clothes dried completely.

The effect of the modifier in these examples is to specify that an endpoint of the scales introduced by the adjectives must be reached (we discuss endpoints in greater detail in the next section). If so, then the difference value has a natural bound-the endpoint itself-which should give rise to a telic interpretation. As shown by (22a)(22b), (21a) and (21b) satisfy tests for telicity.

(22) a. They are straightening the rope completely. $\not$ They have straightened the rope completely.

b. The sun is drying the clothes completely. $\nRightarrow$ The sun has dried the clothes completely.

More complicated cases involve degree modifiers like significantly, which also give rise to telic interpretations, as illustrated by the examples in (23).

(23) a. The independent counsel broadened the investigation significantly.

b. The IC almost broadened the investigation significantly. (AMBIG.)

c. The IC is broadening the investigation significantly. $\nRightarrow$ The IC has broadened the investigation significantly.

Unlike completely, significantly does not make reference to an endpoint of a scale. Such degree modifiers, however, require a particular point to have been reachedfor significantly, whatever counts as significant in the context of utterance. The notion "bounded measure of change" must then be further refined: a telic reading of a DA requires that the difference value specify a lower bound on the degree to which an object must increase in the relevant property over the course of the event. Once this minimal point is reached, the truth conditions for the event description are met.

The modifier significantly is monotone increasing, contrasting with monotone decreasing modifiers such as slightly, which place no lower bound on the degree to which the object must increase in the relevant property (part of a slight increase is also a slight increase). Atelic interpretations of DAs result, as in (24). ${ }^{3}$

(24) a. The independent counsel broadened the investigation slightly.

b. The independent counsel almost broadened the investigation slightly. (UNAMBIGUOUS) 
c. The independent counsel is broadening the investigation slightly. $\Rightarrow$ The independent counsel has broadened the investigation slightly.

This distinction between monotone increasing and decreasing modifiers marks yet another parallel with the mass-count distinction. Mass nouns are also monotone decreasing for quantity: parts of rice are also rice (i.e., the mass noun has cumulative reference (Krifka 1989, 1992)); this property does not hold of count nouns like a plum (a plum does not consist of parts which satisfy the description of a plum), however. What seems to be crucial for deriving telic interpretations in both the mass-count cases and DAs, then, is the identification of some lower bound on the extent to which some object changes as a result of the action described by the verb. We return to a more detailed discussion of these connections in section 4.2.

\subsection{The Scalar Structure of the Base Adjective}

The more complicated types of DA constructions are those in which the interpretation of the difference value is not provided by overt linguistic material, but must somehow be inferred. The bounded/non-bounded distinction has the same effect on telicity in these cases, but boundedness must be determined in other ways. The first set of cases that we consider are those in which information about the scalar structure of the adjectival base plays a crucial role.

As demonstrated by Hay (1998), adjectives fall into two classes according to the structure of the scale onto which they map their arguments. The first class, which we refer to as closed-range adjectives, consists of adjectives that are associated with a scale with a maximal value, where maximality is relative to the adjective's polarity. (For a positive adjective like full, the maximal value corresponds to the upper end of the scale of volume, while for the negative adjective empty, it is the lower end of the scale.) Straight, empty, and dry are examples of such adjectives, the crucial point being that it is in principle possible for something to be so straight/empty/dry that it is cannot be any straighter/emptier/drier. Closedrange adjectives contrast with open-range adjectives like long, wide, and short, for which it is not possible to identify maximal values on the scale. (See Kennedy and McNally this volume for additional discussion of these issues.)

Empirically, the two classes of adjectives are distinguished by the acceptability of modification by certain types of adverbials, such as completely. Assuming that completely makes reference to an endpoint as part of its meaning (see above and also Lehrer 1985), the (un)acceptability of the examples in (25) can be taken as a diagnostic of whether the adjectives fall into the closed- or open-range class.

a. completely straight/empty/dry (closed-range adjectives)

b. ? completely long/wide/short (open-range adjectives)

Although the claim that negative adjectives like short, narrow and slow are openrange may not be immediately intuitive-it seems that the absence of size, width, or speed should count as lower endpoints for the respective scales-the important 
point is that facts like those in (25) indicate that these adjectives utilize scales without maximal values (in the relevant sense). The degrees onto which they map their arguments may asymptotically approach the zero point, but they can never reach it. $^{4} \quad$ These adjectives thus contrast with e.g., flat, empty, and dry, which accept modification by completely, and so must be associated with closed scales.

The open-/closed-range distinction plays a central role in determining the telicity of DAs (Hay 1998). DAs derived from closed-range adjectives typically behave telically, as illustrated in (26) (though see below for qualification). This difference follows naturally from our analysis. Since the scale associated with the adjective has a maximal value, a bound on the difference value can be identified: the measure of change that takes the affected argument to the end of the scale.

(26) a. They are straightening the rope. $\not \Rightarrow$ They have straightened the rope.

b. The clothes are drying. $\nRightarrow$ The clothes have dried.

In contrast, DAs derived from open-range adjectives behave atelically, as demonstrated in (27). Since the scale associated with the adjective lacks a maximal value, there is no basis for determining a bound on the difference value, and the predicate is correctly predicted to be atelic.

(27) a. They are lengthening the rope. $\Rightarrow$ They have lengthened the rope.

b. The snow is slowing. $\Rightarrow$ The snow has slowed.

\subsection{Context-dependent Telicity}

There is a second set of examples in which a bound on the difference value must be inferred: those in which its denotation cannot be identified based on a closed-range base adjective, but instead is determined from other contextual cues. This effect is illustrated in (28). In these examples it is not knowledge about the scalar structure of the adjective that determines the bounded interpretation of the difference value, but rather knowledge about conventional properties of pants and blinds.

(28) a. The tailor lengthened my pants.

b. Kim lowered the blind.

Real-world knowledge informs us that there is a conventional maximal length for pants, and a conventional degree beyond which there is no need for blinds to be further lowered. This knowledge provides a means for the difference value to be assigned a bound. That these sentences are telic is shown by (29).

(29) a. The tailor is lengthening my pants. $\not \Rightarrow$

The tailor has lengthened my pants.

b. Kim is lowering the blind. $\not \Rightarrow$ Kim has lowered the blind.

The examples in (28) contrast with those in (30), which are telic. 
(30) a. The traffic lengthened my commute.

b. Kim lowered the heat.

(31) a. The traffic is lengthening my commute.

$\Rightarrow$ The traffic has lengthened my commute.

b. Kim is lowering the heat. $\Rightarrow \mathrm{Kim}$ has lowered the heat.

Although there is a conventional maximal length for pants, there is no such bounded length for a commute. And while blinds generally have a point at which they cannot be further lowered, heat can, in theory, be lowered indefinitely. Thus, the examples in (28) provide some means for the difference value to be assigned a bound, whereas there is no such information in the sentences in (30). (The examples in (28) recall Dowty's (1979:61) observation that John swam can have a telic interpretation in a context where John is known to swim a set distance every day, as well as the more usual atelic interpretation.)

It is important to note that the identification of the difference value as a bounded measure of change in both the examples discussed in this section and the data in section 3.2 arises through a process of conversational implicature, a process that Olsen $(1994,1997)$ has shown to be at work in other instances of (a)telicity. As shown by (32), the implicature that the change is bounded can be cancelled.

(32) a. The tailor lengthened my pants, but not completely.

b. I straightened the rope, but not completely.

The examples in (32) contrast with those in which the difference value is explicitly supplied by linguistic material. In such instances, the bounded interpretation-and telicity-is not cancellable.

(33) a. \#They straightened the rope completely, but the rope isn't completely straight.

b. \# They widened the road $5 \mathrm{~m}$, but the road didn't increase in width by $5 \mathrm{~m}$.

We suggest that this implicature should be explained in terms of general principles of informativity. Given what we know about tailors, the most informative interpretation of (28a) is the one in which the tailor finished his job. Similarly, what is unique about closed-range adjectives is that the endpoint of the scale is a possible reference point. The most informative interpretation of They straightened the rope then is one in which the rope is straightened completely. In both cases, the effect of the implicature is to impose a completely-like interpretation on the predication, giving rise to the inference of a bounded measure of change. Further evidence that contextually determined telicity arises through implicature comes from the nonredundancy of sentences such as They straightened the rope completely.

The role of conversational implicature in generating telic interpretations of DAs provides an explanation for the adverbial duality facts, first mentioned in the introduction and repeated in (34), which are a hallmark of DAs. 
(34) a. The soup cooled in an hour.

b. The soup cooled for an hour.

These facts have long caused problems for theories of aspectual classification, but they fall out from our analysis. On our account, adverb duality should arise only when the difference value must be inferred, since only then do the principles of conversational implicature come into play. The sentence The soup cooled is most informative on a telic interpretation: the soup cooled to some bounded degree. Such a reading is possible in this example because such a degree is salient, namely room temperature. Since a telic interpretation is possible, the adverbial in (34a) is perfectly acceptable. (Compare (34a) to ?The lake cooled in an hour, which is anomalous because there is no salient bound for the cooling of a lake.) The acceptability of the durative adverbial in (34b), which is felicitous only with atelic predicates, also follows. This example is acceptable precisely because the durative adverbial has the effect of cancelling the telicity implicature. As a result, this sentence receives an interpretation in which the soup cools, but only to some unspecified degree.

The overall analysis is further supported by the observation that the adverbial duality facts disappear when overt linguistic material imposes a bound on the difference value, as completely does in (35).

(35) a. The soup completely cooled in an hour.

b. ?? The soup completely cooled for an hour.

Since telicity does not arise through implicature here, but rather from the truth conditions of the overt material, it cannot be cancelled by the durative adverbial. Instead, its use results in true semantic anomaly.

\subsection{Summary}

To summarize, we have shown that the lexical semantic analysis of DAs presented in section 2 supports a comprehensive and general account of the apparently paradoxical aspectual properties of this class of verbs. The analysis relies crucially on the interaction of linguistic material, the scalar structure of the base adjectives, and extralinguistic knowledge to derive the (a)telicity of a DA as a function of the boundedness of the difference value. The discussion of the role of contextual/pragmatic information in determining telicity is particularly important, as it indicates that (a)telicity cannot be fully specified by the linguistic form, either semantically or, as has more recently been argued, syntactically (e.g., Borer 1998, Slabakova 1997). Our analysis thus provides new insights on the complex interaction between verb meaning, event structure and context in determining the aspectual properties of predicates on different occasions of use. In the next section we show that our analysis can be extended to other types of predicates that have been central to discussions of (a)telicity, and we address in more detail the larger implications of the role of the difference value in determining telicity. 


\section{Consequences of the analysis}

\subsection{Beyond Degree Achievements}

On our account, the interaction of linguistic and contextual factors determines the boundedness of the difference value and hence the telicity of a DA. However, as our account is not tied to idiosyncratic properties of DAs, we predict that the same interaction should come into play in any predicate involving an expression that corresponds to our notion of the difference value. In fact, when we look beyond DAs to other types of predicates that have figured prominently in discussions of (a)telicity, it becomes apparent that contextual as well as linguistic factors play an important role in their analysis as well.

Perhaps the best-known examples in the literature on telicity are those based on verbs of consumption, creation, and motion. Thus, a verb phrase headed by a verb of consumption such as eat the sandwich is a good example of a classically telic predicate; however, the telic interpretation of eat the sandwich actually arises through implicature, since it can be cancelled, as shown by the felicity of (36).

She ate the sandwich but as usual she left a few bites.

As with the examples considered in section 3.3, the telic interpretation is the most informative, and so arises through implicature. It follows that the predicate should display the same adverbial duality facts manifested by DAs. (37) confirms this prediction (see the discussion of (34) above).

a. She ate the sandwich in 5 minutes.

b. She ate the sandwich for 5 minutes.

The examples in (38) and (39) make the same points for two of the other types of predicates commonly cited in discussions of telicity, verbs of motion and verbs of creation. When a bound on the difference value is linguistically specified, a non-cancellable telic interpretation arises. If, however, a bound is only contextually supplied, then the telicity arises through implicature and is cancellable.

(38) a. ?? She ran a mile, but didn't quite finish it.

b. She ran a race, but didn't quite finish it.

(39) a. ?? She drew a $2 \mathrm{~cm}$ line, but it wasn't quite $2 \mathrm{~cm}$ long.

b. She drew a house, but it was missing a door.

Just as DAs have provoked interest as a subset of the verbs of change of state that show both telic and atelic properties, so too there are some verbs of directed motion that show aspectual duality. Levin and Rappaport Hovav (1995) point out there is a subset of the verbs of directed motion whose members are not necessarily telic, but form a class of "atelic verbs of directed motion" (e.g, ascend, descend, 
rise, fall). Furthermore, there are also causative verbs of this type (e.g., lower, raise), though Levin and Rappaport Hovav do not note their existence.

Evidence that such verbs have difference values comes from the fact that they can be overtly specified with measure phrases, as in (40a) and (40b).

(40) a. The plane descended 1000 meters.

b. The water level rose 4 feet.

Given these facts, it is not surprising that like DAs, these verbs of directed motion have both telic and atelic interpretations, as shown in (41). Moreover, telicity may vary with the nature of the affected argument, as in (42).

(41) a. The plane descended in 20 minutes.

b. The plane descended for 20 minutes.

(42) a. The submarine is rising. $\not \Rightarrow$ The submarine has risen.

b. The water level is rising. $\Rightarrow$ The water level has risen.

Our analysis of telicity straightforwardly extends to these facts. Like DAs, these verbs describe a change along a projected scale: the path of movement of the affected argument. Their telicity then should depend on the boundedness of the difference value. In (41), the availability of a maximal value of change (a descent to the ground) should give rise to a telicity implicature. Thus the use of an in-adverbial as in (41a) should be acceptable, and the implicature should be cancellable, as in (41b). Similarly, a maximal measure of change for a submarine's ascent can be identified (ascent to sea level), leading to a telic interpretation of (42a). The water itself has no maximal level; as a result, (42b) gets an atelic interpretation.

\subsection{The Difference Value and the Incremental Theme}

An important consequence of our work is that it suggests a new understanding of the notion "incremental theme," a term introduced by Dowty 1991 (building on Krifka 1989, 1992) to name the argument of a verb whose properties are crucial to the determination of the verb's telicity. This notion was applied by Dowty to certain verbs, primarily verbs of creation and consumption, whose objects, by virtue of their physical extent, define a homomorphism onto the temporal progress of the event denoted by the verb. For instance, in Dowty's 1991 example, mow the lawn, the lawn is the incremental theme since the progress of the entire event can be determined by looking at the state of the lawn. Other types of predicates also figure in discussions of telicity, particularly verbs of change of state and verbs of motion, and attempts have been made to identify homomorphisms between properties of these verbs and the temporal progress of the events they denote in order to account for their telicity (e.g., Jackendoff 1996, Ramchand 1997, Tenny 1987, 1994, Verkuyl 1993). However, distinct types of homomorphisms appear to be necessary for each of the three major verb types. With verbs of change of state, the homomorphism 
is between a gradable property corresponding to the state associated with the verb; with verbs of motion, the homomorphism is defined by the path traversed by one of its arguments.

Although this previous work has sometimes emphasized the distinctiveness of these three lexically-dependent types of telicity, our proposals suggest a more unified analysis (see also Rappaport Hovav and Levin 1999). The major obstacles to a unified analysis have been Dowty's original incremental theme verbs, where most treatments identify the incremental theme argument as playing a central role in determining telicity (e.g., Krifka's 1989, 1992 notion of Mapping to Objects). In contrast, the other two types of telicity are typically analyzed in terms of a change in a property of an argument: a gradable property for verbs of change of state or a position along a path for verbs of motion. In actual fact, the original incremental theme verbs also involve a gradable property, which is defined by the spatial extent of the so-called "incremental theme" argument-characterizable, depending on the verb, in terms of its volume, area, or other relevant spatial dimension. It is for this reason that we described the verb eat in this way in section 1, when we motivated our analysis of DAs. These spatially-defined properties are perhaps less easily dissociable from the arguments themselves than those related to verbs of change of state and verbs of motion, so it is not surprising that these properties have been confused with the arguments. Returning to Dowty's original example, mow the lawn, the true incremental theme, we claim, is not the lawn, but rather its area-a property of the lawn. If this is correct, this case of telicity can be recast in a manner parallel to the other two, as it was in section 1, with a property of an argument again defining the homomorphism. More generally, all three types of telicity can be determined as a function of the boundedness of the difference value defined over a projected scale associated with one of the verb's arguments, where the nature of the scale depends on the lexical meaning of the verb.

Against this background, the semantic object that best corresponds to Dowty's incremental theme is in fact the difference value (i.e., the measure of change along a path of motion, in spatial extent, or in some other scalar property). On this view, the "incremental theme" is properly construed as a measure of some property of an argument of a verb, not an argument, although it may be expressed by an argument-like expression (as in the case of extent objects of some verbs of motion, such as swim the Channel). In fact, Dowty (1991:659) recognizes that with verbs of motion the term "incremental theme" is most appropriately applied to the path of motion, even if it is often left unexpressed, rather than to the argument corresponding to the moving object or theme, which Dowty names the "holistic theme." It would seem reasonable to extend this label to that argument of a verb of consumption, creation, or change of state that stands in a comparable relation to the incremental theme as we have redefined it. The consequence would be the identification of a novel natural class of arguments, in other words, a new semantic role. Several questions follow. Does the holistic theme role receive independent support? If so, is the grammatical behavior of the holistic theme in some way privileged as a consequence of its relationship to the true incremental theme? For instance, could it provide insight into the much debated question of whether aspectual notions figure 
in argument expression, as proposed by Tenny $(1987,1994)$ ? We leave these issues for future research.

Finally, our analysis also clarifies the relationship between telicity and the incremental theme. This relationship is often left unexamined (see e.g., Tenny 1995:68, n. 20), though the implicit assumption seems to be that only telic events have well-defined incremental themes (see e.g., Dowty 1991:568, 607). Our analysis demonstrates that telicity and incremental theme (qua difference value) are independent, however, supporting the proposals in Jackendoff 1996, Krifka 1992, and Ramchand 1997. Any predicate with an associated scale has an incremental theme, but a predicate's telicity depends on whether or not the incremental theme is bounded.

\section{Conclusion}

In this paper, we have argued that the apparently inconsistent aspectual properties of DAs (and related verbs) can be given a uniform analysis which involves the interaction of linguistic material, the inherent scalar structure associated with the base adjective, and extralinguistic knowledge. Our analysis of the DA facts provides a direct parallel with the well-known mass-count distinction, and extends beyond DAs to correctly predict the behavior of related predicates. The analysis has significant implications for models of aspectual representation and provides a better understanding of the interrelations between the semantic notions of telicity and incremental theme.

\section{Endnotes}

* We are grateful to audiences at SALT 9 and USC for very helpful comments on this work. This work was supported in part by NSF Grant SBR-9616453 to Levin. 1. The term "degree achievements" is due to Dowty (1979), who argues that these verbs denote changes of state, and claims that they pattern with achievements on some semantic and syntactic grounds. In this paper, we continue to use the term 'degree achievement', for reasons of historical (in)accuracy, recognizing that there is little evidence that degree achievements are achievements at all. As we show below, in Vendler/Dowty terms, DAs variably display characteristics of accomplishments and activities (Hay 1998).

2. In particular, we remain agnostic as to the best analysis of the causative alternation represented by transitive/intransitive pairs (e.g., Kelly cooled the soup/The soup cooled). If we were to follow Levin and Rappaport Hovav's (1995) lead, for exam-

ple, then we would include a causative element in both the transitive and intransitive forms. On the other hand, other analyses (e.g., Hale and Keyser 1986, Lakoff 1968) argue that the causative component is found only in the transitive form.

3. The indefinite measure phrase $a$ bit is also monotone decreasing, and so gives rise to atelic interpretations of DAs (cf. (18) above): The lake is cooling a bit entails 
The lake has cooled a bit.

4. Cruse (1986:206) touches on this point when he observes that "The value of slow, although it 'tends towards' zero speed, never actually reaches it, but approaches it, as mathematicians say, asymptotically. This is not a physical fact, but a linguistic one: we cannot say completely slow when we mean 'stationary'."

\section{References}

Abusch, D. (1986) "Verbs of Change, Causation, and Time," Report 86-50, CSLI, Stanford University, Stanford, CA.

Bartsch, R. and T. Vennemann (1972) Semantic Structures: A Study in the Relation between Syntax and Semantics, Athenäum, Frankfurt.

Bertinetto, P.M. and M. Squartini (1995) "An Attempt at Defining the Class of 'Gradual Completion' Verbs," in P.M. Bertinetto, V. Bianchi, J. Higginbotham, and M. Squartini, eds., Temporal Reference Aspect and Actionality, 1: Semantic and Syntactic Perspectives, Rosenberg and Sellier, Torino, Italy, 11-26.

Borer, H. (1998) "Passive without Theta Grids," in S.G. Lapointe, D.K. Brentari, and P.M. Farrell, eds., Morphological Interfaces, CSLI Publications, Stanford, CA, 60-99.

Cruse, D.A. (1986) Lexical Semantics, Cambridge University Press, Cambridge.

Declerck, R. (1979) "Aspect and the Bounded/Unbounded (Telic/Atelic) Distinction," Linguistics 17, 761-794.

Dowty, D.R. (1979) Word Meaning and Montague Grammar, Reidel, Dordrecht.

Dowty, D.R. (1991) “Thematic Proto-Roles and Argument Selection,” Language 67, 547-619.

Hale, K.L. and S.J. Keyser (1986) "Some Transitivity Alternations in English", Lexicon Project Working Papers 7, Center for Cognitive Science, MIT, Cambridge, MA.

Hay, J. (1998) "The Non-Uniformity of Degree Achievements," presented at the 72nd Annual Meeting of the LSA, New York, NY.

Jackendoff, R.S. (1996) "The Proper Treatment of Measuring Out, Telicity, and Perhaps Even Quantification in English," Natural Language and Linguistic Theory 14, 305-354.

Kennedy, C. (1997) "Comparison and Polar Opposition," in Proceedings of SALT 7, CLC Publications, Ithaca, NY.

Kennedy, C. (1999) Projecting the Adjective: The Syntax and Semantics of Gradability and Comparison, Garland, New York.

Kennedy, C. and L. McNally (this volume) "From Event Scales to Adjectival Scales: Degree Modification in Deverbal Adjectives".

Krifka, M. (1989) "Nominal Reference, Temporal Constitution and Quantification in Event Semantics," in R. Bartsch, J. van Benthem, and P. van Emde Boas, eds., Semantics and Contextual Expression, Foris, Dordrecht, 75-115.

Krifka, M. (1992) “Thematic Relations as Links between Nominal Reference and 
Temporal Constitution," in I.A. Sag and A. Szabolcsi, eds., Lexical Matters, CSLI Publications, Stanford, CA, 29-54.

Lakoff, G. (1968) "Some Verbs of Change and Causation”, in S. Kuno, ed., Mathematical Linguistics and Automatic Translation, Report NSF-20, Aiken Computation Laboratory, Harvard University, Cambridge, MA.

Lehrer, A. (1985) "Markedness and Antonymy," Journal of Linguistics 21, 397-429.

Levin, B. and M. Rappaport Hovav (1995) Unaccusativity: At the Syntax-Lexical Semantics Interface, MIT Press, Cambridge, MA.

McCawley, J.D. (1976) "Remarks on What Can Cause What," in M. Shibatani, ed., Syntax and Semantics 6: The Grammar of Causative Constructions, Academic Press, New York, 117-129.

Olsen, M.B. (1994) "The Semantics and Pragmatics of Lexical Aspect Features," Studies in the Linguistic Sciences 24, 361-375.

Olsen, M.B. (1997) A Semantic and Pragmatic Model of Lexical and Grammatical Aspect, Garland, New York.

Pustejovsky, J. (1991) “The Syntax of Event Structure," Cognition 41, 47-81.

Ramchand, G.C. (1997) Aspect and Predication, Clarendon Press, Oxford.

Rappaport Hovav, M. and B. Levin (1999) "Two Types of Compositionally Derived Events," unpublished ms., Bar Ilan University and Northwestern University, Ramat Gan, Israel and Evanston, IL.

Rullmann, H. (1995) Maximality in the Semantics of Wh-Constructions, Doctoral dissertation, University of Massachusetts, Amherst.

Seuren, P.A.M. (1978) "The Structure and Selection of Positive and Negative Gradable Adjectives," Papers from the Parasession on the Lexicon, Chicago Linguistic Society, Chicago, IL, 336-346.

Slabakova, R. (1997) “Bulgarian Preverbs: Aspect in Phrase Structure," Linguistics $35,673-704$.

von Stechow, A. (1984a) “Comparing Semantic Theories of Comparison,” Journal of Semantics 3, 1-77.

von Stechow, A. (1984b) “My Reaction to Cresswell's, Hellan's, Hoeksema's, and Seuren's Comments," Journal of Semantics 3, 183-199.

Tenny, C.L. (1987) Grammaticalizing Aspect and Affectedness, Doctoral dissertation, MIT, Cambridge, MA.

Tenny, C.L. (1994) Aspectual Roles and the Syntax-Semantics Interface, Kluwer, Dordrecht.

Tenny, C.L. (1995) "How Motion Verbs are Special: The Interaction of Semantic and Pragmatic Information in Aspectual Verb Meanings," Pragmatics and Cognition 3, 31-73.

Van Valin, R.D., Jr. and R.J. LaPolla (1997) Syntax: Structure, Meaning and Function, Cambridge University Press, Cambridge, UK.

Vendler, Z. (1957) "Verbs and Times," Philosophical Review 56, 143-160.

Verkuyl, H.J. (1972) On the Compositional Nature of the Aspects, Reidel, Dordrecht.

Verkuyl, H. (1993) A Theory of Aspectuality, Cambridge University Press, Cambridge, UK. 